\title{
Ecologia de Paisagens e Licenciamento Ambiental
}

\author{
Landscape Ecology and Environmental Impacts Analysis
}

\author{
Rodrigo Vasconcelos Koblitz', Sílvio José Pereira Júnior', \\ Rafael Cavalcanti de Albuquerque Ajuz ${ }^{1}$ \& Carlos Eduardo Viveiros Grelle ${ }^{2 *}$
}

\author{
${ }^{1}$ Diretoria de Licenciamento Ambiental - Dilic, \\ Instituto Brasileiro do Meio Ambiente e dos Recursos Naturais Renováveis - IBAMA, Brasilia, DF, Brazil \\ ${ }^{2}$ Laboratório de Vertebrados, Departamento de Ecologia, Universidade Federal do Rio de Janeiro - UFRJ, \\ Rio de Janeiro, RJ, Brazil
}

Qual a melhor escala para se desenvolver um estudo na natureza? Para responder a esta pergunta deve-se primeiro pensar qual é a questão a ser respondida. Pensando unicamente nos organismos vivos, a escolha da escala espacial adequada depende da mobilidade dos mesmos, que determinará as dimensões da extensão e o tamanho do grão no estudo (Wiens 1989). Extensão (extent) é a área a ser estudada, considerando-se as populações, enquanto o grão (grain) é o tamanho das unidades de observação, considerando-se os indivíduos a serem amostrados (Wiens 1989). O problema é que alguns padrões na natureza, como a variação espacial da riqueza de espécies, não podem ser explicados por processos em uma única escala espacial e temporal (e.g. Whittaker et al. 2001). A escolha da escala espacial fica ainda mais complicada em estudos multidisciplinares, onde uma resposta precisa ser dada à pergunta (ou perguntas), com fenômenos acontecendo simultaneamente em várias escalas. Qualquer um que tenha participado de um projeto multidisciplinar entende a dificuldade para definir a escala adequada e, seja esta qual for, todas as informações precisam ser espacializadas para viabilizar análises e sínteses.

Estudos com abordagens teóricas e empíricas têm demonstrado a importância da escala espacial (e temporal) para se entender a dinâmica das populações (Hanski \& Gaggiotti 2004) e comunidades (Holyoak et al. 2005). A dispersão de indivíduos entre manchas de hábitat em paisagens heterogêneas é capaz de manter e estruturar as (meta)populações e (meta)comunidades, evidenciando que os estudos desses níveis hierárquicos devem ser feitos em escalas mais amplas do que a local, sendo necessária

\footnotetext{
${ }^{*}$ Send correspondence to: Carlos Eduardo Viveiros Grelle Departamento de Ecologia,

Universidade Federal do Rio de Janeiro - UFRJ, CP 68020, CEP 21941-590, Rio de Janeiro, RJ, Brasil e-mail: grellece@biologia.ufrj.br
}

uma abordagem que considere explicitamente a escala de estudo. Esse é o caso da Ecologia de Paisagens, que enfatiza a importância da escala a ser escolhida pelo observador, de acordo com o processo ou espécie(s) que se pretende estudar (Lorini \& Persson 2001; Metzger 1999, 2001). É uma área do conhecimento que, em sua versão mais contemporânea, tem o objetivo de entender os efeitos da heterogeneidade espacial na ocorrência e manutenção das populações, comunidades e/ou processos, considerando sempre a escala correta a ser estudada. Contudo, originalmente, a Ecologia de Paisagens surgiu na Europa no berço da geografia física e da ciência da vegetação, mas sob grande influência da geografia humana e com foco sobre o planejamento do uso da terra, na vertente conhecida como abordagem geográfica (veja Metzger 2001 e Lorini \& Persson 2001 para uma revisão). Portanto, as distintas abordagens e diferentes interpretações do o que é a Ecologia de Paisagens desde a sua origem denotam a sua capacidade para lidar com questões multidisciplinares, tendo como base a distribuição espacial das informações. De fato, a paisagem pode introduzir a dimensão espacial no desenvolvimento dos estudos, representando ao mesmo tempo objeto e ferramenta de pesquisa, o que permitiria uma articulação entre as diferentes escalas de análise dentro de uma mesma disciplina ou das diversas disciplinas envolvidas, assim como constituiria um fio condutor para a confrontação dos vários pontos de vista disciplinares, inclusive auxiliando a superar as dificuldades relacionadas à disputa entre os especialistas das Ciências Naturais e Sociais e os problemas de comunicação entre os cientistas e os tomadores de decisão (Lorini \& Persson 2001). O próprio conceito de paisagem, caracterizado pela polivalência e polissemia de sentidos, pode ser um instrumento efetivo na criação de métodos e modelos interdisciplinares adequados a situações particulares e práticas, funcionando como um importante conceito diagonal na temática da conservação de biodiversidade (Lorini \& Persson 2001). 
Tendo como foco os aspectos biológicos, a heterogeneidade ambiental é uma das principais causadoras das descontinuidades dos processos evolutivos. A Ecologia de Paisagens é uma área do conhecimento que desde a sua origem tem como objetivo a análise e interpretação dos efeitos da heterogeneidade ambiental (Metzger 2001). Indo além do paradigma da Teoria da Biogeografia de Ilhas (MacArthur \&Wilson 1967), na qual as manchas de habitat são isoladas umas das outras por um entorno homogêneo e inóspito, sendo o tamanho da mancha e o grau de isolamento dessa os fatores determinantes para uma série de processos ecológicos, a Ecologia de Paisagens é uma abordagem que pode levar em consideração a qualidade do entorno (heterogeneidade espacial) e a capacidade de movimentação do organismo estudado (Metzger 2001). Nesse contexto, a paisagem em uma abordagem ecológica é um mosaico de unidades heterogêneas, mais ou menos favoráveis para uma espécie ou a comunidade estudada, dada uma escala de observação (Metzger 2001). Dessa forma, a conectividade na paisagem determina o grau de facilitação ou impedimento de movimentos entre manchas de habitat (e.g. Taylor et al. 2006; Umetsu et al. 2008), sendo a conectividade uma variável chave na estruturação espacial, que pode alterar o fluxo gênico, a capacidade de dispersão das espécies, a viabilidade das populações e os serviços ecossistêmicos (Crooks \& Sanjayan 2006).

A conectividade na paisagem pode ser classificada em dois grupos: estrutural e funcional. A primeira se baseia no aspecto estrutural da paisagem, levando em consideração o arranjo espacial, a densidade, complexidade e qualidade dos corredores de habitat e stepping stones, além da permeabilidade do entorno, enquanto a segunda se refere à resposta comportamental específica de uma espécie aos elementos da paisagem (Metzger 1999; Taylor et al. 2006; Theobald 2006). Devido aos diferentes usos da terra a qualidade da matriz varia na paisagem, sendo a conectividade espécie-específica, já que as espécies apresentam diferentes capacidades de movimentação entre fragmentos (Crouzeilles et al. 2010; Forero-Medina \& Vieira 2007; Taylor et al. 2006; Theobald 2006; Umetsu et al. 2008).

A abordagem de Ecologia de Paisagens vem ganhando força nos processos de licenciamento ambiental, pois é uma forma de integrar as informações para o planejamento e execução de empreendimentos de engenharia, sem ignorar a importância dos processos ecológicos e evolutivos (e.g. Sayer 2009; Pfund 2010; Wu 2006). Conciliar a conservação da biodiversidade, incluindo os processos ecológicos e evolutivos, ao desenvolvimento humano e novos empreendimentos, é um desafio a ser enfrentado. Em países em desenvolvimento e com grande biodiversidade o patrimônio natural é geralmente visto como algo a ser explorado sem planejamento, assim como uma barreira ao desenvolvimento (Meffe \& Carrol 1997). Na verdade os serviços ambientais fornecidos pela natureza são de difícil mensuração, e a sua importância não é amplamente difundida, e por isso a sociedade tem dificuldade de compreender a relação entre biodiversidade e sobrevivência da humanidade (Meffe \& Carrol 1997). Em alguns países como o Brasil mesmo as possíveis vantagens econômicas que o patrimônio natural pode trazer têm sido ignoradas (Medeiros et al. 2011).

No Brasil a viabilidade dos empreendimentos depende do processo de licenciamento ambiental, que é coordenado e fiscalizado por órgãos públicos (federais, estaduais e municipais) e orientado por normas federais (Resoluções Conama nº01/1986 e n 237/1997). A Licença Prévia (LP), concedida na fase preliminar do planejamento do empreendimento ou atividade, aprova sua localização e concepção, atestando a viabilidade ambiental e estabelecendo os requisitos básicos e condicionantes a serem atendidos nas próximas fases de sua implementação. Para empreendimentos e atividades consideradas efetiva ou potencialmente causadoras de significativa degradação do meio ambiente a LP dependerá de Estudo de Impacto Ambiental e respectivo Relatório de Impacto Ambiental (EIA/RIMA), que é avaliado por equipe multidisciplinar. O EIA deve conter minimamente o diagnóstico ambiental dos meios físico, biótico e socioeconômico, incluindo suas interações, análise dos impactos ambientais do projeto e de suas alternativas, definição das medidas mitigadoras dos impactos negativos e elaboração dos programas de acompanhamento e monitoramento dos impactos previstos.

O planejamento do EIA deve ser feito com base científica, incluindo a seleção de amostras e coleta de dados, de modo que toda e qualquer tomada de decisão seja feita baseada em conhecimento científico. Existe um esforço das revistas científicas para geração de conhecimento que possa ser usado para resolver questões ambientais (Diniz-Filho \& Loyola 2010; Milner-Gulland et al. 2009), o que pode resultar em propostas para a gestão de unidades de conservação (e.g. Alexandre et al. 2010), políticas públicas (e.g. Aleixo et al. 2010; Scarano \& Martinelli 2010) e avaliação dos impactos de empreendimentos (e.g. Vasas et al. 2009; Telles et al. 2011). No entanto, nem sempre é simples aplicar o conhecimento científico para resolver questões ambientais fora da academia (e.g. Lima et al. 2010). Os estudos de impacto ambiental são, por essência, multidisciplinares e a espacialização das informações é uma etapa primordial do processo. Neste contexto os estudos de impacto ambiental devem ser feitos considerando os princípios da Ecologia de Paisagens para integrar as informações das vertentes biológicas e sócioambientais em uma única análise (veja uma forma em Sarkar \& Illoldi 2010) e, consequentemente, para verificar a viabilidade ambiental dos empreendimentos.

Embora muitas vezes o empreendimento seja pontual (por exemplo, usinas hidrelétricas e mineração), os impactos ambientais gerados podem se estender pela paisagem no entorno e, frequentemente, esta extensão só será conhecida após a fase de monitoramento. O mapeamento das unidades da paisagem e o inventário primário são fundamentais e, como já exposto no início deste artigo, a extensão e o grão são dependentes de cada empreendimento. Pensando nos 
fundamentos básicos da Ecologia de Paisagens, o ideal é trabalhar com uma abordagem multidisciplinar, que considere fatores ambientais e sócioeconômicos, além de amostrar diferentes unidades da paisagem para se ter uma noção da heterogeneidade ambiental, o que será resultado da escala de observação escolhida. Pensando no poder de inferência estatística, quanto maior for a diversidade $\beta$ (sensu Whittaker 1960), ou seja, a heterogeneidade ambiental, maior a necessidade do número de réplicas na paisagem. Pensando nas amostragens e abordagens biológicas, envolvendo indivíduos de espécies diferentes, análises de detectabilidade (MacKenzie et al. 2005) são recomendáveis para se perceber as falsas ausências que podem mudar completamente um cenário esperado após a implementação de um empreendimento.

Em decorrência da heterogeneidade ambiental e das diferenças de composição de espécies de uma área para outra, análises baseadas em conectividade na paisagem são importantes para priorizar fragmentos em escalas regionais, enquanto que em escalas de extensão geográfica mais ampla, os algoritmos de complementariedade são mais adequados para priorizar a escolha de uma área em detrimento de outra. Estudos realizados com vertebrados no Cerrado (e.g. Pinto et al. 2007) e na Mata Atlântica (e.g. Pinto \& Grelle 2009), e com vegetação na Mata Atlântica (Grelle et al. 2010), têm demonstrado a utilidade da complementariedade na escolha, por exemplo, de áreas para serem unidades de conservação.

Na prática, tem-se aventado recentemente no licenciamento ambiental federal a relevância da discussão da complementariedade para o debate da viabilidade ambiental e para a proposição de medidas mitigadoras e compensatórias, muitas vezes representadas como preservação de áreas. O que se propõe é a definição de quais as espécies e processos ecológicos são exclusivos dos ambientes em que se pretendem empreendimentos com potencial de degradação. Frente ao conceito de complementaridade, o foco seria a procura por ambientes que possam contribuir de forma semelhante aos que serão degradados, podendo manter processos ecológicos semelhantes. Quanto às espécies, em alguns casos, deve ser considerado mais relevante preservar áreas que somam biodiversidade, tendo em mente a complementariedade, do que aquelas com maior riqueza separadamente. Essa decisão deverá levar em conta a importância de cada espécie e não somente a quantidade, indicando, por exemplo, a relevância de características funcionais das espécies (diversidade funcional). Existe então a incorporação do raciocínio do Planejamento Sistemático da Conservação - PSC
(Pressey et al. 1993), para o processo de licenciamento. Deve-se, entretanto, observar as diferenças nas aplicações. No clássico PSC procura-se identificar as áreas mais relevantes para a conservação de uma dada região. Identifica-se o que se quer conservar (alvos) e atribui-se o quanto se quer conservar de cada alvo (metas). Ainda é possível preservar locais mais próximos um do outro do que locais distantes e ainda se pretende, prioritariamente, conservar locais que tenham um menor valor econômico, que de um modo geral são menos ameaçados pela ocupação humana.

No licenciamento ambiental, em relação ao meio biótico, a definição de alvos para discussão da viabilidade ambiental proposta deve incorporar os seguintes critérios (Tabela 1):

- Caso 1 - Espécies que só ocorrem dentro da área que será degradada e com média/alta detectabilidade. Dentro desse critério também se encontram as espécies que só foram coletadas dentro da área que será degradada, mas da qual vários indivíduos foram coletados, sugerindo uma elevada abundância local em um ambiente que será suprimido pelo empreendimento;

- Caso 2 - Espécies que ocorrem dentro e fora da área que será degradada, mas com baixas abundâncias no entorno, levantando questões quanto à viabilidade das populações remanescentes;

- Caso 3 - Áreas com maior quantidade de unicatas na área que será degradada. São as espécies que foram coletadas com apenas um indivíduo, ou seja, em apenas uma ocasião do estudo. Sozinhas não sugerem, indicam ou explicam quase nada, pois se foram encontradas apenas uma vez, não se pode dizer que não existam em outro local, mas apenas que não foram coletadas, devido à baixa detectabilidade. Portanto, o fato de não as termos encontrado em outro local pode ser apenas um artefato da amostragem. Muitas vezes são constituídas por espécies raras, que podem indicar a relevância de um ambiente.

Sobre todas as categorias de espécies discutidas acima, aquelas que figuram nas listas de espécies ameaçadas ou ainda são insuficientemente conhecidas para serem enquadradas em algum grau de ameaça, devem ser observadas com cuidado. Aquelas espécies ameaçadas que só foram encontradas no entorno e com baixa detectabilidade devem ser observadas com prioridade, pois significa que tais espécies podem ser ausências falsas na área que será degradada e só não foram

Tabela 1. Critérios de seleção de alvos proposto para que seja utilizado na discussão de complementariedade no licenciamento ambiental. Todos esses critérios são discutidos em termos de relevância, dependendo também do grau de ameaça da espécie e/ou processo ecológico. Veja o texto para explicação dos Casos 01, 02 e 03.

\begin{tabular}{lcc}
\multicolumn{1}{c}{ Ocorrência } & Critério de seleção dos alvos & Hipótese \\
\hline Espécies que ocorram somente dentro da área que será degradada & Média e/ou alta detectabilidade & Caso 01 \\
Espécies que ocorram dentro e fora da área que será degradada & Baixa abundância no entorno & Caso 02 \\
Área que será degradada & Rica em unicatas & Caso 03 \\
\hline
\end{tabular}


encontradas devido às baixas abundâncias. Não há nenhuma equação definida de sistema de pesos a se atribuir a essas espécies e devemos considerá-las em cada caso sem um roteiro pré-definido. Igualmente, a prioridade de análise dos alvos não é pré-definida, pois deve ser considerado que todos têm igual importância.

Quando os alvos são processos ecológicos, uma decisão é a escolha de "ambientes" como substitutos desses processos, como tem sido proposto pela Environmental Protect Agency - EPA, órgão ambiental dos EUA (US Environmental Protection Agency Office of Federal Activities 1999). No caso do licenciamento ambiental, os alvos escolhidos orientam as áreas a serem preservadas. $\mathrm{O}$ que se procura com essa análise é a relação de causa-consequência entre o impacto do empreendimento e sua mitigação e compensação. Quando se atribui ao empreendimento os impactos que ele promove, baseados em espécies e processos ecológicos, encontra-se uma relação objetiva e racional para a avaliação de impactos. Uma vez definidos esses alvos, deve-se refletir sobre a escala de análise. O objetivo focado em espécies traz diferentes situações de decisão. Em alguns casos, poderia ser aceita a extinção de uma espécie em escala local, na bacia hidrográfica, mas talvez aceitar a extinção da espécie no bioma seja um preço com que a sociedade não estaria disposta a arcar.

A abordagem de Ecologia de Paisagens no licenciamento ambiental é relevante para a decisão de viabilidade e para as ações de mitigação e compensação de um empreendimento quando existirem alvos definidos. A seleção de alvos, representados na Tabela 1, é uma informação oriunda das amostragens de biodiversidade. Tal informação é útil, não só intrinsecamente em termos das espécies, mas também para qualificar a heterogeneidade da paisagem, o que orientará as tomadas de decisões.

No que se refere à utilização espacial da região onde se insere o empreendimento, as medidas de mitigação e compensação devem ter ao menos um componente espacialmente explícito em suas decisões. Desse modo, a espacialização das informações coletadas em campo e as análises pertinentes, serão balizadores para a decisão de viabilidade e a adequação do empreendimento ao menor impacto possível.

Enfim, a abordagem em Escala de Paisagem, buscando entender como a biota e as pessoas estão distribuídas em relação à heterogeneidade ambiental, parece ser adequada para estudos feitos durante o processo de licenciamento ambiental, sendo um exemplo da aplicação da ciência em outros setores da sociedade.

\section{Agradecimentos}

Maria Lucia Lorini pela oportunidade, Renato Crouzeilles pelos comentários em uma versão preliminar, e Maria Lucia Lorini e José Alexandre F. Diniz-Filho por vários comentários na versão final. William E. Magnusson, Albertina
Lima e Flávia R. C. Costa por sugestões e discussões sobre planejamento sistemático e as unicatas. C. E. V Grelle agradece ao CNPq pelos auxílios e bolsa de produtividade e a FAPERJ pelo auxílio Jovem Cientista do Estado do Rio de Janeiro.

\section{Referências}

Aleixo A et al., 2010. Mudanças climáticas e a biodiversidade dos biomas brasileiros: passado, presente e futuro. Natureza \& Conservação, 8:194-196. http://dx.doi.org/10.4322/ natcon.00802016

Alexandre B, Crouzeilles R \& Grelle CEV, 2010. How can we estimate buffer zones of protected areas? A proposal using biological data. Natureza \& Conservação, 8:165-170. http:// dx.doi.org/10.4322/natcon.00802010

Crooks KR \& Sanjayan M, 2006. Connectivity Conservation. Cambridge University Press. Cambridge. http://dx.doi. org/10.1017/СBO9780511754821

Crouzeilles R, Lorini ML \& Grelle CEV, 2010. Deslocamento na matriz para espécies da Mata Atlântica e a dificuldade da construção de perfis ecológicos. Oecologia Australis, 14:875903. http://dx.doi.org/10.4257/oeco.2010.1404.06

Diniz-Filho JAF \& Loyola RD, 2010. Conservation Science in Brazil: Challenges for the $21^{\text {st }}$ Century. Natureza \& Conservação, 8:1-3. http://dx.doi.org/10.4322/ natcon.00801000

Forero-Medina G \& Vieira MV, 2007. Conectividade funcional e a importância da interação organismo-paisagem. Oecologica Brasiliensis, 11:493-502. http://dx.doi.org/10.4257/ oeco.2007.1104.03

Grelle CEV, Lorini ML \& Pinto MP, 2010. Reserve selection based on vegetation in the Brazilian Atlantic forest. Natureza \& Conservação, 8:46-53. http://dx.doi.org/10.4322/ natcon.00801007

Hanski I \& Gaggiotti OE, 2004. Ecology, Genetics, and Evolution of Metapopulations. Elsevier Academic Press, Burlington.

Holyoak M, Leibold MA \& Holt RD, 2005. Metacommunities: Spatial Dynamics and Ecological Communities. Chigaco University Press, Chicago.

Lima FP, Muniz JR \& Marco Junior P, 2010. Evaluating Brazilian conservation projects: the weak link between practice and theory. Natureza \& Conservação, 8:41-45. http://dx.doi. org/10.4322/natcon.00801006

Lorini ML \& Persson VG, 2001. A paisagem: um conceito diagonal entre as ciências geográficas e biológicas e um instrumento para a ciência transdisciplinar da biodiversidade. Revista de Estudos Ambientais, 3:5-19.

MacArthur RH \& Wilson EO, 1967. The Theory of Island Biogeography. Princeton: Princeton University Press.

Mackenzie, D. et al., 2005. Occupancy estimation and modeling: inferring patterns and dynamics of species occurrence. New York: Academic Press.

Medeiros R, Young CEF, Pavese HB \& Araújo FFS, 2011. Contribuição das Unidades de Conservação para a Economia Nacional. Brasília: UNEP-WCMC. 
Meffe GK \& Carroll CR, 1997. Principles of Conservation Biology. New York: Sinauer Associates.

Milner-Gulland EJ, Fisher M, Browne S, Redford KH, Spencer M \& Sutherland WJ, 2009. Do we need to develop a more relevant conservation literature? Oryx, 44:1-2. http://dx.doi. org/10.1017/S0030605309991001

Metzger JP, 1999 Estrutura da Paisagem e Fragmentação: análise Bibliográfica. Anais da Academia Brasileira de Ciências, 71:445-463.

Metzger JP, 2001. O que é Ecologia de Paisagens? Biota Neotropica, 1:1-9.

Pinto MP \& Grelle CEV 2009. Reserve selection and persistence: Complementing the existing Atlantic Forest reserve system. Biodiversity and Conservation, 18:957-968.

Pinto MP, Mathias PVC, Blamires D et al 2007. Selecting priority areas to conserve Psittacines in the Brazilian cerrado: minimizing human-conservation conflicts. Bird Conservation International, 17:13-22. http://dx.doi. org/10.1017/S0959270906000578

Pfund J-L, 2010. Landscape-scale research for conservation and development in the tropics: fighting persisting challenges. Current Opinion in Environmental Sustainability, 2:117-126. http://dx.doi.org/10.1016/j.cosust.2010.03.002

Pressey RL, Humphries CJ, Margules, CR, Vane-Wright, RI \& Williams PH, 1993. Beyond Opportunism: Key Principles for Systematic Reserve Selection. Trends in ecology and Evolution, 8:124-128. http://dx.doi. org/10.1016/0169-5347(93)90023-I

Sarkar S \& Illoldi-Rangel P, 2010. Systematic conservation planing: an updated protocol. Natureza \& Conservação, 8:1926. http://dx.doi.org/10.4322/natcon.00801003

Sayer J, 2009. Reconciling conservation and development: Are landscape the answer? Biotropica, 41:649-652. http:// dx.doi.org/10.1111/j.1744-7429.2009.00575.x

Scarano FR \& Martinelli G, 2010. Brazilian list of threatened plant species: reconciling scientific uncertainty and political decision-making. Natureza \& Conservação, 8:13-18. http:// dx.doi.org/10.4322/natcon.00801002
Taylor PD, Fahrig L \& With K, 2006 Landscape connectivity: a return to the basics. In: Crooks KR \& Sanjayan M (eds) Connectivity Conservation. Cambridge: Cambridge University Press. p. 29-43, http://dx.doi.org/10.1007/s10980-009-9346-8

Telles MPC et al. 2011. A geographical genetics framework for inferring homing reproductive behavior in fishes. Genetica, 139:243-253. http://dx.doi.org/10.1007/ s10709-010-9541-1

Theobald DM, 2006 Exploring the functional connectivity of landscapes using landscape networks. In: Crooks KR \& Sanjayan M (eds). Connectivity Conservation. Cambridge: Cambridge University Press. p. 416-443. http://dx.doi. org/10.1017/CBO9780511754821.019

Umetsu F, Metzger JP \& Pardini R, 2008 Importance of estimating matrix quality for modeling species distribution in complex tropical landscapes: a testt with Atlantic forest small mammals. Ecography, 31:359-370. http://dx.doi. org/10.1111/j.0906-7590.2008.05302.x

US Environmental Protection Agency Office of Federal Activities. 1999. Considering Ecological Processes in Environmental Impact Assessments. http://www.epa.gov/ oecaerth/resources/policies/nepa/ecological-processeseia-pg.pdf

Vasas V, Magura T, Jordán F \& Tóthmérés B, 2009. Graph theory in action: evaluating planned highway tracks based on connectivity measures. Landscape Ecology, 24:581-586. http://dx.doi.org/10.1007/s10980-009-9346-8

Wiens J, 1989. Spacial scales in ecology. Functional Ecology, 3:385-397.

Whittaker RH, 1960. Vegetation of the Siskiyou Mountains, Oregon and California. Ecological Monographs, 30:279-338. http://dx.doi.org/10.2307/1943563

Whittaker RJ, Willis KJ \& Field R, 2001. Scale and species richness: towards a general, hierarchical theory of species diversity. Journal of Biogeography, 28:453-470. http://dx.doi. org/10.1046/j.1365-2699.2001.00563.x

Wu J, 2006. Landscape ecology, cross-disciplinarity, and sustainability science. Landscape Ecology, 21:1-4. http:// dx.doi.org/10.1007/s10980-006-7195-2

Recebido: Outubro de 2011

Primeiro Decisão: Outubro de 2011 Aceito: Novembro de 2011 